\title{
Attention impairment in bipolar disorder: a systematic review
}

\author{
Evelyn V. M. Camelo ${ }^{1}$, Bruna Velasques ${ }^{1}$, Pedro Ribeiro ${ }^{1}$, Tania Netto ${ }^{1}$, Elie Cheniaux ${ }^{1,2}$ \\ 1. Universidade Federal do Rio de Janeiro, Rio de Janeiro, RJ, Brazil \\ 2. Universidade do Estado do Rio de Janeiro, Rio de Janeiro, RJ, Brazil
}

\begin{abstract}
Bipolar disorder (BD) has been associated with marked cognitive impairment, including euthymic periods. Attention is among the most compromised functions in BD. Changes related to learning, memory, and visuospatial abilities can be derived from these attention impairments. The objective of this article is to review the scientific literature on the performance of BD patients in attention tests. A systematic review was performed of controlled studies that assessed attention in patients diagnosed with BD aged between 18 and 65 years. The databases included Medline, LILACS, Cochrane Library, Institute for Scientific Information Web of Knowledge, and Scientific Electronic Library Online (SciELO), and the search encompassed the period from 2008 to 2013. Only studies that had a minimum sample of 10 patients were included. A total of 110 articles fulfilled the inclusion criteria. Compared with healthy control subjects, bipolar patients showed poorer attention performance. Compared with other mental disorders, BD was associated with poorer performance than unipolar depression but better performance than schizophrenia. When bipolar patients in different phases of the disease were compared with one another, the performance of euthymic patients was similar to or better than patients in a depressive state; moreover, manic patients performed worse than depressive patients. Attention is significantly impaired in BD. Attention impairment in BD is milder than in schizophrenia but greater than in unipolar depression. Attention impairment is possibly more severe in manic and depressed episodes than in euthymic periods.Keywords: attention, cognition, neuropsychological tests, bipolar disorder.
\end{abstract}

Received 20 April 2013; received in revised form 20 August 2013; accepted 21 August 2013. Available online 23 December 2013.

\section{Introduction}

Cognitive deficits were first studied in brain injury and dementia and later in schizophrenia. However, since the 1970s, much effort has been devoted to understanding cognitive function in mood disorders, particularly bipolar disorder (BD; Caligiuri, \& Ellwanger, 2000). The literature has provided evidence of stable and persistent cognitive impairments across the phases of $\mathrm{BD}$, including euthymia, particularly in the following domains: sustained attention, learning, memory, visuospatial ability, and executive function (Caligiuri, \& Ellwanger, 2000; Latalova, Jan, Tomas, Dana, \& Hana, 2011). In BD, attentional changes are very relevant and can affect other cognitive functions,

Evelyn V. M. Camelo, Bruna Velasques, Pedro Ribeiro, and Elie Cheniaux, Universidade Federal do Rio de Janeiro, Instituto de Psiquiatria, Rio de Janeiro, RJ, Brazil. Tania Netto, Universidade Federal do Rio de Janeiro, Faculdade de Medicina, Departamento de Radiologia, Rio de Janeiro, RJ, Brazil. Elie Cheniaux, Universidade do Estado do Rio de Janeiro, Faculdade de Ciências Médicas, Rio de Janeiro, RJ, Brazil. Correspondence regarding this article should be directed to: Evelyn Camelo, Rua Ambrosina 100/202, Tijuca, 20540-120, Rio de Janeiro, RJ, Brazil. Phone: +55 (21) 94723522. E-mail: evypsi@gmail.com such as memory, learning, and executive function (Goodwin, Jaminson, \& Ghaemi, 2007).

Numerous review articles have been published on cognitive impairments in $\mathrm{BD}$, but these studies did not specifically address attention impairments (Sachs, Schaffer, \& Winklbaur, 2007; Latalova et al., 2011; Kałwa, 2010; Quaishi \& Frangou, 2002). To our knowledge, only one review article published 9 years ago (Clark, \& Goodwin, 2004) focused specifically on attentional changes in bipolar patients. The present study provides an update on the knowledge of attention impairments in patients with $\mathrm{BD}$ by systematically reviewing controlled studies published in the past 5 years.

\section{Methods}

We performed a systematic review of the scientific literature on attention impairments in BD. References were identified in five databases (i.e., Medline, Institute for Scientific Information [ISI] Web of Knowledge, Cochrane Library, Scientific Electronic Library Online [SciELO], and LILACS) from 2008 to May, 27, 2013. The following search terms were used: "bipolar" and "attention" or "neuropsychological" or "cognition" or "cognitive." The inclusion criteria were the following: samples with at least 10 patients diagnosed with $\mathrm{BD}$, aged 18 and 65 years, and evaluations that used 
neuropsychological tests that assessed attention. Only controlled trials (e.g., comparisons with healthy controls, with other mental disorders, or among BD patients in different affective states) were considered. Review articles, case reports, letters to the editor, and book chapters were excluded. No search of unpublished work was performed. Citations within a paper were also included as an additional source of references.

\section{Results}

The initial search retrieved 7,885 citations from Medline, 7396 from the ISI Web of Knowledge, 328 from Cochrane Library, 113 from SciELO, and 148 from LILACS, with some overlap among the databases. A total of 879 abstracts were classified as potentially relevant according to our criteria. After the appraisal of the full-text articles, 110 citations were selected.

\section{Studies that compared BD patients with healthy control subjects}

As shown in Table 1, 91 studies compared patients with a diagnosis of $\mathrm{BD}$ with healthy control subjects. In 80 of these studies, bipolar patients performed significantly worse in attention tests compared with healthy controls. In 11 studies, however, no significant difference was found between groups. In 53 of the 91 studies, the patients were not differentiated according to their mood state. In 36 of the 38 studies that incorporated such discrimination, both euthymic patients and patients in a manic or depressed state performed worse on attention tests than controls. One of these 38 studies found no significant difference between euthymic bipolar patients and healthy controls. Among the 80 studies in which the bipolar patients had poorer results compared with controls, sustained attention was evaluated in 40 studies, divided attention was evaluated in 42 studies, and selective attention was evaluated in 18 studies. Some of the studies evaluated more than one modality of attention.

\section{Studies that compared BD with other mental disorders}

As shown in Table 2, 15 studies compared BD patients with other mental disorders. In ten of these studies, comparisons were made with schizophrenia. The performance of bipolar patients on attention tests was significantly superior in seven of the ten studies. Among these seven studies, sustained attention was evaluated in four studies, and divided attention was evaluated in three studies. In two studies that evaluated divided and selective attention, no significant differences were found between the two groups. In only one study, patients with schizophrenia outperformed bipolar patients. In this study, sustained attention was assessed.

Five studies compared bipolar patients with unipolar depression patients. Sustained attention was evaluated in four of these studies. Selective attention was evaluated in two of these studies, and divided attention was evaluated in one of these studies. Unipolar depression patients had better attention performance in four of the five studies. No significant differences were found between the two groups in one study in measures of sustained and selective attention.

Studies that compared the different phases of BD

As seen in Table 3, four studies compared BD patients in different mood states. In two studies that evaluated sustained attention, euthymic bipolar patients were contrasted with patients in a depressed state. In one of these studies, euthymic patients had superior performance on attention tests, whereas no significant differences were found between groups in the other study. In the other two studies, depressive bipolar patients performed better than manic patients on tests that evaluated divided and selective attention.

\section{Discussion}

The present systematic review described controlled studies with bipolar patients who underwent neuropsychological evaluation using attention tests. Our aim was to provide an update on the body of knowledge about attention impairment in patients with BD based on studies published over the past five years.

According to our review, compared with health controls, patients with BD presented worse performance. Recently, other reviews that compared BD patients with healthy controls with regard to cognitive performance have been published (Thompson et al., 2005; Borges, Trentini, Bandeira, \& DellÁglio, 2008; Burdick, Gunawardane, Goldberg, Halperin, Garno, \& Malhotra, 2007; Rocca \& Lafer, 2006). Our results on attention impairment in BD were very similar to those found in these reviews. These studies showed that cognitive impairment is part of the neuropsychological profile of BD patients. Specifically, these findings showed that these individuals have impairments in attention performance compared with healthy controls, regardless of whether the patients are euthymic, manic, or depressed. For example, Bora, Yücel, \& Pantelis (2010) reviewed 12 studies that had samples with at least 10 subjects in each group, including patients with BD and healthy individuals, which were neurocognitively compared. All of these studies showed that patients with BD had inferior performance in tasks that measure executive function, memory, and attention.

According to our review, in seven of the ten studies that compared BD with schizophrenia, bipolar patients performed better in attention tests. Indeed, most of the review articles identified in the present study showed that patients cognitive deficits are more pronounced in patients with schizophrenia than in patients with BD in several cognitive functions, including psychomotor speed, verbal and visual memory, attention, and executive function (Goldberg et al., 1993; Evans, Heaton, Paulsen, McAdams, Heaton, \& Jeste, 1999; Hawkins, Hoffman, Quinlan, Rakfeldt, Docherty, \& 
Table 1. Studies that compared bipolar patients with normal controls with regard to attention performance.

\begin{tabular}{|c|c|c|c|c|}
\hline Study & Sample and design & Instruments & Type of attention & Results \\
\hline Trivedi, Goel, Sharma, Singh, \& Tandon, 2008 & $15 \mathrm{BD}$-e $v s .15 \mathrm{NC}$ & CPT & Sustained attention & $\mathrm{BD}=\mathrm{NC}$ \\
\hline $\begin{array}{l}\text { Morisano, Wing, Sacco, Arenovich, \& Goerge, } \\
2013\end{array}$ & $16 \mathrm{BD} v s .17 \mathrm{NC}$ & $\mathrm{CPT}$ & Sustained attention & $\mathrm{BD}=\mathrm{NC}$ \\
\hline Gualtieri \& Morgan, 2008 & $96 \mathrm{BD}$ vs. $907 \mathrm{NC}$ & SCWT, CPT, SAT & $\begin{array}{l}\text { Selective, sustained, divided } \\
\text { attention }\end{array}$ & $\mathrm{BD}=\mathrm{NC}$ \\
\hline Frantom, Allen, \& Cross, 2008 & $19 \mathrm{BD} v s .19 \mathrm{NC}$ & SCWT, CPT, TMT & $\begin{array}{l}\text { Selective, sustained, divided } \\
\text { attention }\end{array}$ & $\mathrm{BD}=\mathrm{NC}$ \\
\hline Aydemir \& Ender, 2009 & $38 \mathrm{BD}$ vs. $19 \mathrm{NC}$ & SCWT & Selective attention & $\mathrm{BD}=\mathrm{NC}$ \\
\hline Antila et al., 2009 & $39 \mathrm{BD} v s .55 \mathrm{NC}$ & Digit Span, TMT & Divided attention & $\mathrm{BD}=\mathrm{NC}$ \\
\hline Torralva et al., 2011 & $15 \mathrm{BD}$ vs. $15 \mathrm{NC}$ & Digit Span, TMT & Divided attention & $\mathrm{BD}=\mathrm{NC}$ \\
\hline Tuulio-Henriksson et al., 2011 & $17 \mathrm{BD}$ vs. $66 \mathrm{NC}$ & Digit Span & Divided attention & $\mathrm{BD}=\mathrm{NC}$ \\
\hline Xu et al., 2012 & $223 \mathrm{BD}$ vs. $202 \mathrm{NC}$ & Digit Span & Divided attention & $\mathrm{BD}=\mathrm{NC}$ \\
\hline Wu et al., 2011 & $40 \mathrm{BD} v s .19 \mathrm{NC}$ & Digit Symbol & Divided attention & $\mathrm{BD}=\mathrm{NC}$ \\
\hline $\begin{array}{l}\text { Li et al., } 2012 \\
\text { Burdick et al.,2011 } \\
\text { Schretlen et al.,2013 }\end{array}$ & $\begin{array}{l}34 \mathrm{BD} v s .17 \mathrm{NC} \quad 80 \\
\mathrm{BD}-\mathrm{e} v s .149 \mathrm{NC} \\
\text { vs. } 340 \mathrm{NC}\end{array}$ & $\begin{array}{l}\text { Go/No-Go } \\
\text { CPT }\end{array}$ & $\begin{array}{l}\text { Divided attention Sus- } \\
\text { tained attention } \\
\text { Sustained attention }\end{array}$ & $\begin{array}{l}\mathrm{BD}=\mathrm{NC} \\
\mathrm{BD}<\mathrm{NC} \\
\mathrm{BD}<\mathrm{NC}\end{array}$ \\
\hline Sanchez-Moreno et al., 2009 & $65 \mathrm{BD}$ vs. $35 \mathrm{NC}$ & Digit Span, TMT & Divided attention & $\mathrm{BD}<\mathrm{NC}$ \\
\hline $\begin{array}{l}\text { Martino, Strejilevich, Torralva, \& Manes, } 2010 \\
\text { Elshahawi,Essawi,Rabie,Mansour,Beshry,\&M } \\
\text { ansour,2011 }\end{array}$ & $\begin{array}{l}85 \mathrm{BD}-\mathrm{e} v s .34 \mathrm{NC} \\
100 \mathrm{BD}-\mathrm{e} v s .50 \mathrm{NC}\end{array}$ & $\begin{array}{l}\text { Digit Span, TMT } \\
\text { TMT }\end{array}$ & $\begin{array}{l}\text { Divided attention } \\
\text { Divided attention }\end{array}$ & $\begin{array}{l}\mathrm{BD}<\mathrm{NC} \\
\mathrm{BD}<\mathrm{NC}\end{array}$ \\
\hline $\begin{array}{l}\text { Normala, Abdul, Azlin, Nik, Hazli, \& Shah, } \\
2010\end{array}$ & $40 \mathrm{BD}$ vs. $40 \mathrm{NC}$ & Digit Span, TMT & Divided attention & $\mathrm{BD}<\mathrm{NC}$ \\
\hline Burdick et al.,2011 & $103 \mathrm{BD}$-e vs. $35 \mathrm{NC}$ & Digit Span, TMT & Divided attention & $\mathrm{BD}<\mathrm{NC}$ \\
\hline Torrent et al., 2011 & $79 \mathrm{BD}-\mathrm{e} v s .35 \mathrm{NC}$ & Digit Span, TMT & Divided attention & $\mathrm{BD}<\mathrm{NC}$ \\
\hline Martino et al., 2011a & $87 \mathrm{BD}-\mathrm{e} v s .39 \mathrm{NC}$ & Digit Span, TMT & Divided attention & $\mathrm{BD}<\mathrm{NC}$ \\
\hline Martino et al., 2011b & $81 \mathrm{BD}-\mathrm{e} v s .34 \mathrm{NC}$ & Digit Span, TMT & Divided attention & $\mathrm{BD}<\mathrm{NC}$ \\
\hline Bonnín et al., 2012 & $103 \mathrm{BD}$-e vs. $30 \mathrm{NC}$ & Digit Span, TMT & Divided attention & $\mathrm{BD}<\mathrm{NC}$ \\
\hline Torrent et al., 2012 & $68 \mathrm{BD}-\mathrm{e} v s .45 \mathrm{NC}$ & Digit Span, TMT & Divided attention & $\mathrm{BD}<\mathrm{NC}$ \\
\hline Pattanayak, Sagar, \& Mehta, 2012 & $30 \mathrm{BD}-\mathrm{e} v s .20 \mathrm{NC}$ & Digit Span, TMT & Divided attention & $\mathrm{BD}<\mathrm{NC}$ \\
\hline Martino et al., 2008 & $50 \mathrm{BD} v s .30 \mathrm{NC}$ & $\begin{array}{l}\text { Digit Span, TMT, } \\
\text { CPT }\end{array}$ & Divided, sustained attention & $\mathrm{BD}<\mathrm{NC}$ \\
\hline $\begin{array}{l}\text { Soeiro-de-Souza, Machado-Vieira, Soares Bio, } \\
\text { Do Prado, \& Moreno, } 2012\end{array}$ & $66 \mathrm{BD} v s .78 \mathrm{NC}$ & $\begin{array}{l}\text { Digit Span, TMT, } \\
\text { SCWT }\end{array}$ & Divided, selective attention & $\mathrm{BD}<\mathrm{NC}$ \\
\hline Soeiro-de-Souza et al., 2012 & $72 \mathrm{BD}$ vs. $76 \mathrm{NC}$ & $\begin{array}{l}\text { Digit Span, TMT, } \\
\text { SCWT }\end{array}$ & Divided, selective attention & $\mathrm{BD}<\mathrm{NC}$ \\
\hline Hill et al., 2009 & $22 \mathrm{BD} v s .41 \mathrm{NC}$ & Digit Span, CPT & Divided, sustained attention & $\mathrm{BD}<\mathrm{NC}$ \\
\hline Jabben, Krabbendam, \& van Os, 2009 & $76 \mathrm{BD}$ vs. $61 \mathrm{NC}$ & Digit Span, CPT & Divided, sustained attention & $\mathrm{BD}<\mathrm{NC}$ \\
\hline $\begin{array}{l}\text { Thompson, Gray, Crawford, Hughes, Young, \& } \\
\text { Ferrier, } 2009\end{array}$ & $63 \mathrm{BD}$-e vs. $63 \mathrm{NC}$ & $\begin{array}{l}\text { Digit Span, CPT, TMT } \\
\text { SCWT, COWA, TL }\end{array}$ & $\begin{array}{l}\text { Divided, sustained, selective } \\
\text { attention }\end{array}$ & $\mathrm{BD}<\mathrm{NC}$ \\
\hline Mur et al., $2008 b$ & $15 \mathrm{BD}$ vs. $15 \mathrm{NC}$ & Digit Span, SCWT & Divided, selective attention & $\mathrm{BD}<\mathrm{NC}$ \\
\hline Simonsen et al., 2008 & $73 \mathrm{BD}$ vs. $124 \mathrm{NC}$ & Digit Span & Divided attention & $\mathrm{BD}<\mathrm{NC}$ \\
\hline Barrett et al., 2009 & $32 \mathrm{BD}-\mathrm{m} v s .67 \mathrm{NC}$ & Digit Span & Divided attention & $\mathrm{BD}<\mathrm{NC}$ \\
\hline Gogos, Joshua, \& Rossell, 2010 & $40 \mathrm{BD}$ vs. $43 \mathrm{NC}$ & Digit Span & Divided attention & $\mathrm{BD}<\mathrm{NC}$ \\
\hline $\begin{array}{l}\text { Vaskinn, Sundet, Simonsen, Hellvin, Melle, \& } \\
\text { Andreassen, } 2011\end{array}$ & $106 \mathrm{BD}$ vs. $340 \mathrm{NC}$ & Digit Span & Divided attention & $\mathrm{BD}<\mathrm{NC}$ \\
\hline Yates, Dittmann, Kapczinski, \& Trentini, 2011 & $65 \mathrm{BD}$ vs. $34 \mathrm{NC}$ & Digit Span & Divided attention & $\mathrm{BD}<\mathrm{NC}$ \\
\hline Dickerson et al., 2011 & $60 \mathrm{BD}$ vs. $312 \mathrm{NC}$ & Digit Span & Divided attention & $\mathrm{BD}<\mathrm{NC}$ \\
\hline Gerber et al., 2012 & $30 \mathrm{BD}$-e vs. $20 \mathrm{NC}$ & Digit Span & Divided attention & $\mathrm{BD}<\mathrm{NC}$ \\
\hline Chou et al., 2012 & $23 \mathrm{BD}$-e vs. $33 \mathrm{NC}$ & Digit Span & Divided attention & $\mathrm{BD}<\mathrm{NC}$ \\
\hline $\begin{array}{l}\text { Soeiro-de-Souza, Bio, Dias, Vieta, Macha- } \\
\text { do-Vieira, \& Moreno, } 2013\end{array}$ & $109 \mathrm{BD}$ vs. $96 \mathrm{NC}$ & Digit Span & Divided attention & $\mathrm{BD}<\mathrm{NC}$ \\
\hline Burdick et al., 2009 & $24 \mathrm{BD}$ vs. $24 \mathrm{NC}$ & SCWT & Selective attention & $\mathrm{BD}<\mathrm{NC}$ \\
\hline Pompei et al., 2011 & $39 \mathrm{BD}$-e $v s .48 \mathrm{NC}$ & SCWT & Selective attention & $\mathrm{BD}<\mathrm{NC}$ \\
\hline Lewandowski et al., 2011 & $31 \mathrm{BD}$ vs. $20 \mathrm{NC}$ & SCWT & Selective attention & $\mathrm{BD}<\mathrm{NC}$ \\
\hline $\begin{array}{l}\text { Juselius, Kieseppa, Kaprio, Lonnqvist, \& Tuu- } \\
\text { lio-Henriksson, } 2009\end{array}$ & $26 \mathrm{BD}$-e vs. $114 \mathrm{NC}$ & TMT & Divided attention & $\mathrm{BD}<\mathrm{NC}$ \\
\hline Solé et al., 2012 & $43 \mathrm{BD}$ vs. $42 \mathrm{NC}$ & TMT & Divided attention & $\mathrm{BD}<\mathrm{NC}$ \\
\hline Chang et al., 2012 & $94 \mathrm{BD}$ vs. $29 \mathrm{NC}$ & TMT & Divided attention & $\mathrm{BD}<\mathrm{NC}$ \\
\hline Benson et al., 2008 & $30 \mathrm{BD} v s .66 \mathrm{NC}$ & $\mathrm{CPT}$ & Sustained attention & $\mathrm{BD}<\mathrm{NC}$ \\
\hline Mur et al., 2008a & $33 \mathrm{BD}$ vs. $33 \mathrm{NC}$ & CPT & Sustained attention & $\mathrm{BD}<\mathrm{NC}$ \\
\hline Tabarés-Seisdedos et al., 2008 & $43 \mathrm{BD}$ vs. $25 \mathrm{NC}$ & $\mathrm{CPT}$ & Sustained attention & $\mathrm{BD}<\mathrm{NC}$ \\
\hline
\end{tabular}




\begin{tabular}{|c|c|c|c|c|}
\hline Study & Sample and design & Instruments & Type of attention & Results \\
\hline $\begin{array}{l}\text { Malloy-Diniz, Neves, Abrantes, Fuentes, \& } \\
\text { Correa, } 2009\end{array}$ & $39 \mathrm{BD}$ vs. $53 \mathrm{NC}$ & CPT & Sustained attention & $\mathrm{BD}<\mathrm{NC}$ \\
\hline Strakowski et al., 2009 & $70 \mathrm{BD}-\mathrm{m} v s .34 \mathrm{NC}$ & $\mathrm{CPT}$ & Sustained attention & $\mathrm{BD}<\mathrm{NC}$ \\
\hline Lahera et al., 2009 & $24 \mathrm{BD}$-e $v s .38 \mathrm{NC}$ & $\mathrm{CPT}$ & Sustained attention & $\mathrm{BD}<\mathrm{NC}$ \\
\hline Swann, Lijffijt, Lane, Steinberg, \& Moeller, 2009 & $112 \mathrm{BD}$ vs. $71 \mathrm{NC}$ & $\mathrm{CPT}$ & Sustained attention & $\mathrm{BD}<\mathrm{NC}$ \\
\hline Sanchez-Morla et al., 2009 & $73 \mathrm{BD}$-e vs. $67 \mathrm{NC}$ & $\mathrm{CPT}$ & Sustained attention & $\mathrm{BD}<\mathrm{NC}$ \\
\hline $\begin{array}{l}\text { Brooks, Bearden, Hoblyn, Woodard, \& Ketter, } \\
2010\end{array}$ & $16 \mathrm{BD}$-e $v s .11 \mathrm{NC}$ & $\mathrm{CPT}$ & Sustained attention & $\mathrm{BD}<\mathrm{NC}$ \\
\hline $\begin{array}{l}\text { Van der Wer-Eldering, Burger, Holthausen, } \\
\text { Aleman, \& Nolen, } 2010\end{array}$ & $110 \mathrm{BD}$ vs. $75 \mathrm{NC}$ & $\mathrm{CPT}$ & Sustained attention & $\mathrm{BD}<\mathrm{NC}$ \\
\hline Ancín et al., 2011 & 143 BD-e vs. $101 \mathrm{NC}$ & $\mathrm{CPT}$ & Sustained attention & $\mathrm{BD}<\mathrm{NC}$ \\
\hline $\begin{array}{l}\text { Van der Wer-Eldering, Burger, Jabben, } \\
\text { Holthausen, Aleman, \& Nolen, } 2011\end{array}$ & $108 \mathrm{BD}$ vs. $75 \mathrm{NC}$ & $\mathrm{CPT}$ & Sustained attention & $\mathrm{BD}<\mathrm{NC}$ \\
\hline Arts et al., 2011 & $76 \mathrm{BD}$ vs. $61 \mathrm{NC}$ & $\mathrm{CPT}$ & Sustained attention & $\mathrm{BD}<\mathrm{NC}$ \\
\hline Pan, Hsieh, \& Liu, 2011 & $32 \mathrm{BD}$-e vs. $39 \mathrm{NC}$ & $\mathrm{CPT}$ & Sustained attention & $\mathrm{BD}<\mathrm{NC}$ \\
\hline Howells, Ives-Keliperi, Horn, \& Stein, 2012 & $12 \mathrm{BD}$-e $v s .9 \mathrm{NC}$ & $\mathrm{CPT}$ & Sustained attention & $\mathrm{BD}<\mathrm{NC}$ \\
\hline Sepede et al., 2012 & $24 \mathrm{BD}$-e $v s .24 \mathrm{NC}$ & $\mathrm{CPT}$ & Sustained attention & $\mathrm{BD}<\mathrm{NC}$ \\
\hline Donohoe et al., 2012 & $110 \mathrm{BD}$ vs. $163 \mathrm{NC}$ & $\mathrm{CPT}$ & Sustained attention & $\mathrm{BD}<\mathrm{NC}$ \\
\hline Fleck et al., 2012 & $50 \mathrm{BD}$ vs. $34 \mathrm{NC}$ & $\mathrm{CPT}$ & Sustained attention & $\mathrm{BD}<\mathrm{NC}$ \\
\hline $\begin{array}{l}\text { Lee, Altshuler, Glahn, Miklowitz, Ochsner, \& } \\
\text { Green, } 2013\end{array}$ & $68 \mathrm{BD} v s .36 \mathrm{NC}$ & $\mathrm{CPT}$ & Sustained attention & $\mathrm{BD}<\mathrm{NC}$ \\
\hline Cummings et al., 2013 & $125 \mathrm{BD}$ vs. $171 \mathrm{NC}$ & СРT & Sustained attention & $\mathrm{BD}<\mathrm{NC}$ \\
\hline Strakowski et al., 2010 & $108 \mathrm{BD}-\mathrm{m} v s .48 \mathrm{NC}$ & DSCPT & Sustained attention & $\mathrm{BD}<\mathrm{NC}$ \\
\hline Hellvin et al., 2012 & $55 \mathrm{BD}-\mathrm{m}$ vs. $110 \mathrm{NC}$ & DSCPT & Sustained attention & $\mathrm{BD}<\mathrm{NC}$ \\
\hline Holmes et al., 2008 & $65 \mathrm{BD}-\mathrm{d} v s .52 \mathrm{NC}$ & RVIP & Sustained attention & $\mathrm{BD}<\mathrm{NC}$ \\
\hline Roiser et al., 2009 & $49 \mathrm{BD}-\mathrm{d} v s .55 \mathrm{NC}$ & RVIP & Sustained attention & $\mathrm{BD}<\mathrm{NC}$ \\
\hline Maalouf et al., 2010 & $52 \mathrm{BD}$ vs. $28 \mathrm{NC}$ & RVIP & Sustained attention & $\mathrm{BD}<\mathrm{NC}$ \\
\hline Yoram et al., 2013 & $47 \mathrm{BD}$ vs. $31 \mathrm{NC}$ & RVIP & Sustained attention & $\mathrm{BD}<\mathrm{NC}$ \\
\hline Vierck et al., 2013 & $96 \mathrm{BD}$ vs. $24 \mathrm{NC}$ & RVIP & Sustained attention & $\mathrm{BD}<\mathrm{NC}$ \\
\hline Chaves et al., 2011 & $29 \mathrm{BD}$ vs. $30 \mathrm{NC}$ & IP-CPT, Digit Span & Sustained, divided attention & $\mathrm{BD}<\mathrm{NC}$ \\
\hline Mora, Portella, Forcada, Vieta, \& Mur, 2012 & $28 \mathrm{BD}$-e vs. $26 \mathrm{NC}$ & $\begin{array}{l}\text { CPT, Digit Span, } \\
\text { SCWT }\end{array}$ & $\begin{array}{l}\text { Sustained, divided, selective } \\
\text { attention }\end{array}$ & $\mathrm{BD}<\mathrm{NC}$ \\
\hline Iverson et al., 2011 & $43 \mathrm{BD}$ vs. $659 \mathrm{NC}$ & CPT, SCWT & Sustained, selective attention & $\mathrm{BD}<\mathrm{NC}$ \\
\hline Levy, 2013 & 30 BD-e vs. $22 \mathrm{NC}$ & CPT, SCWT & Sustained, selective attention & $\mathrm{BD}<\mathrm{NC}$ \\
\hline Iverson et al., 2009 & $47 \mathrm{BD}$ vs. $47 \mathrm{NC}$ & CPT, SCWT, SAT & $\begin{array}{l}\text { Sustained, selective, divided, } \\
\text { attention }\end{array}$ & $\mathrm{BD}<\mathrm{NC}$ \\
\hline Martinez-Aran et al., 2008 & 65 BD-e vs. $35 \mathrm{NC}$ & TMT, SCWT & Divided, selective attention & $\mathrm{BD}<\mathrm{NC}$ \\
\hline López-Jaramillo et al., 2010b & 40 BD-e vs. $20 \mathrm{NC}$ & TMT, SCWT & Divided, selective attention & $\mathrm{BD}<\mathrm{NC}$ \\
\hline Marshall et al., 2012 & $256 \mathrm{BD}$ vs. $97 \mathrm{NC}$ & TMT, SCWT & Divided, selective attention & $\mathrm{BD}<\mathrm{NC}$ \\
\hline $\begin{array}{l}\text { Doğanavşargil, Bokmen, Akbas, Cinemre, } \\
\text { Metin, \& Karaman, } 2013\end{array}$ & 60 BD-e vs. $20 \mathrm{NC}$ & TMT, SCWT & Divided, selective attention & $\mathrm{BD}<\mathrm{NC}$ \\
\hline López-Jaramillo et al., 2010a & 98 BD-m vs. $66 \mathrm{NC}$ & TMT, SCWT, CT & Divided, selective attention & $\mathrm{BD}<\mathrm{NC}$ \\
\hline Torres et al., 2010 & $45 \mathrm{BD}$ vs. $25 \mathrm{NC}$ & $\begin{array}{l}\text { TMT, SCWT, RVIP, } \\
\text { CVLT-II }\end{array}$ & $\begin{array}{l}\text { Divided, selective, sustained } \\
\text { attention }\end{array}$ & $\mathrm{BD}<\mathrm{NC}$ \\
\hline Cuesta et al., 2011 & $65 \mathrm{BD}$ vs. $76 \mathrm{NC}$ & TMT, Digit Span Digit & Divided attention & $\mathrm{BD}<\mathrm{NC}$ \\
\hline Watson et al.,2012 & $\mathrm{BD}-\mathrm{d} v s .55 \mathrm{NC}$ & Symbol & Divided attention & $\mathrm{BD}<\mathrm{NC}$ \\
\hline Pradhan, Chakrabarti, Nehra, \& Mankotia, 2008 & $48 \mathrm{BD}$-e vs. $23 \mathrm{NC}$ & PGIMS & Selective attention & $\mathrm{BD}<\mathrm{NC}$ \\
\hline Wobrock et al., 2009 & $18 \mathrm{BD} v s .23 \mathrm{NC}$ & TMT & Divided attention & $\mathrm{BD}>\mathrm{NC}$ \\
\hline Mahlberg, Adli, Bschor, \& Kienast, 2008 & $28 \mathrm{BD}-\mathrm{m}$ vs. $30 \mathrm{BD}-\mathrm{d}$ & TMT & Divided attention & $\mathrm{BD}>\mathrm{NC}$ \\
\hline Liu, Chen, Hsieh, Su, Yeh, \& Chen, 2010 & $27 \mathrm{BD} v s .21 \mathrm{NC}$ & TAP & Divided attention & $\mathrm{BD}<\mathrm{NC}$ \\
\hline Shan et al., 2011 & $69 \mathrm{BD} v s .22 \mathrm{NC}$ & Digit Symbol & Sustained attention & $\mathrm{BD}<\mathrm{NC}$ \\
\hline
\end{tabular}

>, better performance; <, worse performance; =, similar performance. BD, bipolar disorder; NC, normal control; SZ, schizophrenia; UP, unipolar depression; BD-e, bipolar disorder, euthymic; BD-d, bipolar disorder, depressive; BD-m, bipolar disorder, manic; TMT, Trail Making Test; CPT, Continuous Performance Test ; CPTII, Continuous Performance Test II, version 5; PCTP, Penn Continuous Performance Test; CPT-IP, Continuous Performance Test, identical pairs version; DS-CPT, Degraded Stimulus Continuous Performance Test; CPT-AX, Continuous Performance Test-AX (a character or number preceded by another character or number as a target); RVIP, Rapid Visual Information Processing; SCWT, Stroop Color Word Test; CT, Cancellation Test; SAT, Shifting Attention Test; CB, conditioned blocking; CVLT-II, California Verbal Learning Test; TAP, Attentional Performance Test; TL, Tower of London; COWA, Controlled Oral Word Association Test; PGIMS, PGI memory scale; PASAT, Paced Auditory Serial Addition. 
Table 2. Studies that compared attention performance in bipolar disorder and other mental disorders.

\begin{tabular}{|c|c|c|c|c|}
\hline Study & Sample and design & Instrument & Type of attention & Results \\
\hline Gogos et al., 2009 & $12 \mathrm{BD}$-e $v s .28 \mathrm{SZ}$ & Digit Span & Divided attention & $\mathrm{BD}>\mathrm{SZ}$ \\
\hline Barret et al., 2009 & $32 \mathrm{BD}-\mathrm{m} v s .44 \mathrm{SZ}$ & Digit Span, CB & Divided attention & $\mathrm{BD}>\mathrm{SZ}$ \\
\hline Cuesta et al., 2011 & $65 \mathrm{BD} v s .86 \mathrm{SZ}$ & Digit Span, TMT & Divided attention & $\mathrm{BD}>\mathrm{SZ}$ \\
\hline Tabarés-Seisdedos et al., 2008 & $43 \mathrm{BD}$ vs. $47 \mathrm{SZ}$ & Asarnow CPT & Sustained attention & $\mathrm{BD}>\mathrm{SZ}$ \\
\hline $\begin{array}{l}\text { Lee et al., } 2013 \\
\text { Schretlen et al.,2013 }\end{array}$ & $\begin{array}{l}68 \mathrm{BD} \text { vs. } 38 \mathrm{SZ} \\
126 \mathrm{BD} \text { vs. } 110 \mathrm{SZ}\end{array}$ & $\begin{array}{l}\text { CPT } \\
\text { CPT }\end{array}$ & $\begin{array}{l}\text { Sustained attention Sustained } \\
\text { attention }\end{array}$ & $\begin{array}{l}\mathrm{BD}>\mathrm{SZ} \\
\mathrm{BD}>\mathrm{SZ}\end{array}$ \\
\hline Cummings et al., 2013 & $125 \mathrm{BD} v s .573 \mathrm{SZ}$ & $\mathrm{CPT}$ & Sustained attention & $\mathrm{BD}>\mathrm{SZ}$ \\
\hline Pradhan et al., 2008 & $48 \mathrm{BD}-\mathrm{e} v s .32 \mathrm{SZ}$ & PGIMS & Selective attention & $\mathrm{BD}=\mathrm{SZ}$ \\
\hline Wobrock et al., 2009 & $18 \mathrm{BD}$ vs. $24 \mathrm{SZ}$ & TMT & Divided attention & $\mathrm{BD}=\mathrm{SZ}$ \\
\hline Donohoe et al., 2012 & $110 \mathrm{BD}$ vs. $487 \mathrm{SZ}$ & $\mathrm{CPT}$ & Sustained attention & $\mathrm{BD}<\mathrm{SZ}$ \\
\hline Benson et al., 2008 & $30 \mathrm{BD}$ vs. $34 \mathrm{UP}$ & $\mathrm{CPT}$ & Sustained attention & $\mathrm{BD}<\mathrm{UP}$ \\
\hline Iverson et al., 2011 & $43 \mathrm{BD}$ vs. $143 \mathrm{UP}$ & CPT, SCWT & Sustained, selective attention & $\mathrm{BD}<\mathrm{UP}$ \\
\hline Xu et al., 2012 & $223 \mathrm{BD}$ vs. $293 \mathrm{UP}$ & Digit Span & Divided attention & $\mathrm{BD}<\mathrm{UP}$ \\
\hline Maalouf et al., 2010 & $\begin{array}{l}18 \text { BD-e vs. } 14 \text { BD-d vs. } \\
20 \text { UP }\end{array}$ & RVIP & Sustained attention & $\begin{array}{l}\mathrm{BD}-\mathrm{e}<\mathrm{UP} \text { BD-d } \\
<\mathrm{UP}\end{array}$ \\
\hline Gualtieri \& Morgan, 2008 & $96 \mathrm{BD} v s .285 \mathrm{UP}$ & CPT, SCWT & Sustained, selective attention & $\mathrm{BD}=\mathrm{UP}$ \\
\hline
\end{tabular}

See Table 1 for abbreviations

Table 3. Studies that compared attention performance in the different phases of bipolar disorder.

\begin{tabular}{lllll}
\hline Study & Sample and design & Instruments & Types of attention & Results \\
\hline Maalouf et al., 2010 & 18 BD-e $v$ s. 14 BD-d & RVIP & Sustained attention & BD-e $=$ BD-d \\
Van der Wer-Eldering et al., 2011 & 45 BD-e $v$ s. 63 BD-d & CPT & Sustained attention & BD-e $>$ BD-d \\
Mahlberg et al., 2008 & 28 BD-m $v$ s. 30 BD-d & TMT & Divided attention & BD-d $>$ BD-m \\
Soeiro-de-Souza et al., 2012 & 41 BD-m vs. 25 BD-d & Digit Span, TMT, SCWT & Divided, selective attention & BD-d $>$ BD-m \\
\hline
\end{tabular}

See Table 1 for abbreviations

Sledge, 1997; Rocca \& Lafer, 2006; Quaishi \& Frangou, 2002). For example, Daban et al. (2006) selected 38 studies that compared $\mathrm{BD}$ and schizophrenia patients during the execution of neuropsychological tasks. Patients with BD performed better on tasks that measured Intelligence Quotient (IQ), attention, memory, and executive function. The authors proposed that the poor performance in schizophrenia patients occurred because of the presence of psychotic symptoms, the duration of the illness, and hospitalization. Simonsen et al. (2008) found that BD patients with psychotic symptoms have similar performance as schizophrenia patients in some neurocognitive tasks, such as verbal memory and processing speed. Some authors (Andreasen, \& Powers, 1974; Strauss, Bohannon, Stephens, \& Pauker, 1984; Goldberg et al., 1993; Evans et al., 1999) believe that defining the mood state in samples of BD patients is important because manic patients perform worse than depressive or euthymic patients in working memory, selective attention, and divided attention tasks, and manic patients perform similarly to schizophrenia patients.

We found only five studies that compared BD patients with unipolar depression patients by applying attention tests. In four of these studies, BD patients had worse performance (Benson et al., 2008; Xu et al., 2012; Maalouf et al., 2010). According to other review articles (Rocca \& Lafer., 2006; Murphy \& Sahakian, 1999), patients with BD present worse performance than unipolar depression patients not only in attention tests but also in several other cognitive tasks, such as executive function and working memory.

Our search found only four studies that compared BD patients in different mood states (i.e., euthymia, mania, and depression). In two studies, manic patients presented worse performance than depressed patients. However, the distinction between depressed and euthymic patients was less evident. Two articles (Martínez-Arán et al., 2009; Rocca \& Lafer, 2006) showed that euthymic patients had difficulty in cognitive tasks, but this impairment was less severe compared with depressive and manic patients. Some review articles (Van Gorp, Altshuler, Theberge, Wilkins, \& Dixon, 1998; Cavanagh, Van Beck, Muir, \& Blackwood, 2002; Clark, Iversen, \& Goodwin, 2002; Deckersback, McMurrich, Ogutha, Savage, Sachs, \& Rauch, 2004) reported consistent deficits in sustained attention, verbal memory, and executive function in mania. Moreover, patients with BD in the depressive or euthymic phase performed better than manic patients in attention tasks (Zubieta, Huguelet, O'Neil, \& Giordani, 2001; Clark et al., 2002; Thompson et al., 2005; Xu et al., 2012), verbal learning (Clark et al., 2002; Deckersbach et al., 2004), and visual memory (MacQueen \& Young, 2003; Deckersbach et al., 2004).

One hypothesis that may explain the worse performance in manic patients in sustained attention tests is related to the typical impulsivity exhibited by these patients. They answer quickly and incorrectly 
before the stimulus appears, thus impairing their performance. Impairments in sustained attention were observed in the Continuous Performance Test (CPT), which represents a central neuropsychological deficit associated with mania (Murphy et al., 1999).

Attention is a complex system related to the activation of other cognitive functions; thus, attention impairments in BD patients can be primarily related to other cognitive dysfunctions (Van Gorp et al., 1998; Martínez-Arán et al., 2009). In BD, alterations in attention are highly relevant and can affect other cognitive functions, such as learning, executive function, and memory (Goodwin et al., 2007).

Cognitive deficits become worse over the course of BD (Vieta, 2012; Levy et al., 2009) and are associated with a greater number of disease episodes (Vieta et al., 2012). Selva et al. (2007) failed to find differences between psychotic and non-psychotic subjects on a series of memory, executive function, and attention tests. Harkavy-Friedman et al. (2006) found that suicide attempters with BD had worse performance than nonsuicidal bipolar patients in psychomotor performance, working memory, attention, and impulse control.

Patients with BD generally exhibit typical cognitive development premorbidly but exhibit deficits by the first episode that are amplified as the symptoms worsen. Some data suggest that cognitive deficits may precede the onset of mania; therefore, identifying cognitive predictors of bipolar disorder would be beneficial to facilitate early intervention (Lewandowski, Cohen, Keshavan, \& Ongür, 2011; Olvet, Burdick, \& Cornblatt, 2013).

First-episode mania patients were found to have less neurocognitive deficits in psychomotor speed, attention, learning and memory, executive function, and IQ compared with multiple-episode patients (Hellvin et al., 2012; Van Gorp et al., 1998).

Psychiatric medications commonly used in BD can affect cognition. According to some review articles (Honig, Arts, Ponds, \& Riedel, 1999; Pachet, \& Wisniewski, 2003), lithium may exert mild negative effects in tasks of verbal memory and psychomotor speed, whereas visuo-spatial performance, attention, and executive performance are spared. Lithium has also been shown to exert a neuroprotective effect and be related to better cognitive performance in patients with BD (Bauer, Alda, Priller, \& Young, 2003). Atypical antipsychotics have shown more negative effects on cognition compared with lithium and anticonvulsants (Arts, Jabben, Krabbendam, \& van Os, 2011; Macqueen, \& Young, 2003; Torrent et al., 2011; Yurgelun-Todd et al., 2002).

Some studies have reported that cognitive impairment in patients with BD represents a trait marker of the disease (Clark et al., 2004). These studies have proposed a neurodegenerative hypothesis to explain the cognitive deficits associated with BD (McKinnon, Cusi, \& Macqueen, 2012). Cognitive damage would be an endophenotype of the disorder and a marker associated with this mental disorder. The term "endophenotype" was used by Gottesman (2003) to describe a trait that may be intermediate on the chain of causality from genes to diseases. Some family relatives of affected patients also carry the endophenotype, although not the disorder phenotype (i.e., affective symptoms) in the case of BD (Adida et al., 2012). In fact, some studies also described attention deficits in unaffected relatives of individuals with mood disorders (Bora, Yucel, \& Pantelis, 2009; Brotman, Rooney, Skup, Pine, \& Leibenluft, 2009; Grunebaum, Cohler, Kauffman, \& Gallant, 1978; Klimes-Dougan, Ronsaville, Wiggs, \& Martinez, 2006; Zalla et al., 2004).

Gottesman, \& Gould (2003) discussed endophenotypes and suggested five criteria that should be characteristic of a trait to qualify it as an endophenotype. These five criteria are used to assess the viability of using measures of neuropsychological dysfunction as endophenotypes for genetic studies of BD (Savitz, Solnes, \& Ramesar, 2005). The importance of early interventions in BD have been extensively studied, and recent efforts have been made to identify individuals who are at increased risk; e.g., relatives of bipolar patients (Bora et al., 2009; Olvet et al., 2013). For this reason, some researchers have recently begun to focus on the genetic contributions to discrete (as opposed to global) cognitive processes, such as executive function, working memory, and attention. For example, a version of executive function evaluated by selective and sustained attention tests, e.g., WCST and CPT (Conners, 2000) was reported to have a degree of heritability (Savitz et al., 2005).

To demonstrate that a trait is an endophenotype, the trait must be shown to be mood state-independent and heritable (Gottesman, \& Gould, 2003). Thus, studies that examine neurocognitive aspects and neuroanatomic changes, together with genetics studies, are important to improve our understanding of the neural basis of BD (Kurnianingsih, Kuswanto, McIntyre, Qiu, Ho, \& Sim, 2011).

Considering the neuroanatomic changes that occur in $\mathrm{BD}$, a correlation has been found between a longer disease duration and more pronounced atrophy in the frontal cortex, an area that is closely related to attention (Stoll, Renshaw, Yurgelun-Todd, \& Cohen, 2000; Kemptom, Geddes, Ettinger, Williams, \& Grasby, 2008). Studies of bipolar patients tested attentional impairment using the CPT and structural and functional magnetic resonance imaging and reported changes in the dorsolateral prefrontal cortex (Rocca \& Lafer, 2006), prolonged amygdala overactivation, and prefrontal cortex atrophy (Sax, Strakowski, Zimmerman, DelBello, Keck, \& Hawkins, 1999). In addition to these findings, other studies have reported that certain neuroanatomic structures are associated with attention dysfunction during mania (Sax et al., 1999). One such structure is the right prefrontal cortex, which appears to be involved in sustained attention (Manly, \& Robertson, 1997). Furthermore, functional neuroimaging studies of bipolar patients have detected activation in the right prefrontal cortex during the 
assessment of sustained attention (Coull, Frith, Frackowiak, \& Grasby, 1996; Paus, Zatorre, \& Hofle, 1997). Other modalities of attention, such as selective and divided attention, have also been associated with functional or structural alterations. In patients with $\mathrm{BD}$, a reduction of neural responsiveness was observed in regions involved in selective attention within the posterior and inferior parietal lobes (Pompei et al., 2011). Additionally, impairment in divided attention in patients with bipolar depression has been attributed to a reduction of attentional resources by the central executive (i.e., the working memory component) and impaired activation in the frontal lobe (Paus, Zatorre, $\&$ Hofle, 1997). According to the results of the present review, all types of attention are significantly impaired in BD (Ancín et al., 2011; Andersson, Barder, Hellvin, Løvdahl, \& Malt, 2008; Barrett, Mulholland, Cooper, \& Rushe, 2009; Bonnín et al., 2012; Burdick et al., 2009; Iverson, Brooks, \& Young, 2009). Specifically, sustained attention and divided attention are more severe in mania and in depression, respectively (Murphy et al., 1999).

Attentional impairment in BD is less severe than in schizophrenia but greater than in unipolar depression and possibly more severe in the mania and depression phases of BD than in the euthymia phase. These findings have prompted us to propose the development of cognitive rehabilitation techniques for individuals with $\mathrm{BD}$ that are similar to those used for persons with frontal lobe dysfunction (Levine, Turner, \& Stuss, 2008) or brain injury (Ponsford, 2008). Attention is directly related to other cognitive functions, such as learning, memory, and executive function. Alterations in these and other cognitive functions could at least partially derive from attention deficits. Further studies are needed to investigate the attention alterations in $\mathrm{BD}$, especially longitudinal studies that would allow an enhanced understanding of the progressive character of attention deficits in $\mathrm{BD}$.

\section{Role of funding source}

None.

\section{Conflict of interest}

None of the authors has any conflicts of interest to disclose.

\section{References}

Adida, M., Azorin, J. M., Fakra, E., Belzeaux, R., Kaladjian, A., Pomietto, P., \& Corréard, N. (2012). Schizophrenia and/or bipolar disorder: neurobiological endophenotypes. Encephale, 38(Suppl. 3), S98-S102.

Ancín, I., Cabranes, J. A.,Santos, J. L., Sánchez-Morla, E. M., Rodríguez-Moya L, Pousada-Casa, A., Fernández, C., Aparicio, A., Barabash, A. (2011). CHRNA7 haplotypes are associated with impaired attention in euthymic bipolar disorder. Journal of affective disorders, 133(1-2), 340-345.

Andersson, S., Barder, H. E., Hellvin, T., Løvdahl, H., \& Malt, U. F. (2008). Neuropsychological and electrophysiological indices of neurocognitive dysfunction in bipolar II disorder. Bipolar Disorder, 10(8), 888-899

Andreasen, N. J. C., \& Powers, P. S. (1974). Overinclusive thinking in mania and schizophrenia. British Journal of Psychiatry, 125, 452-456.
Antila, M., Partonen, T., Kieseppä, T., Suvisaari, J., Eerola, M., Lönnqvist, J., \& Tuulio-Henriksson, A. (2009). Cognitive functioning of bipolar I patients and relatives from families with or without schizophrenia or schizoaffective disorder. Journal of Affective Disorders, 116(1-2), 70-79.

Arts, B., Jabben, N., Krabbendam, L., \& van Os, J. (2011). A 2-year naturalistic study on cognitive functioning in bipolar disorder. Acta Psychiatrica Scandinavica, 123(3), 190-205.

Aydemir, O., \& Ender, K. (2009). What does the subjective assessment of cognitive functioning measure in bipolar disorder? Correlation with the objective assessment of cognitive functioning. Turk Psikiyatri Dergisi, 20(4), 332-338.

Barrett, S. L., Mulholland, C. C., Cooper, S. J., \& Rushe, T. M. (2009). Patterns of neurocognitive impairment in first-episode bipolar disorder and schizophrenia. British Journal of Psychiatry, 195(1), 67-72.

Bauer, M., Alda, M., Priller, J., \& Young, L. T. (2003). Implications of the neuroprotective effects os lithium for the treatment of bipolar and neurodegenerative disorders. Pharmacopsychiatry, 36(Suppl. 3), S250-S254

Benson, B. E., Willis, M. W., Ketter, T. A., Speer, A., Kimbrell, T. A., George, M. S., Herscovitch, P., \& Post, R. M. (2008). Interregional cerebral metabolic associativity during a continuous performance task (Part II): differential alterations in bipolar and unipolar disorders. Psychiatric Research, 164(1), 30-47.

Bonnín, C. M., Sánchez-Moreno, J., Martínez-Arán, A., Solé, B., Reinares, M., Rosa, A. R., Goikolea, J. M., Benabarre, A., Ayuso-Mateos, J. L., Ferrer, M., Vieta, E., \& Torrent, C. (2012). Subthreshold symptoms in bipolar disorder: impact on neurocognition, quality of life and disability. Journal of Affective Disorders, 136(3), 650-659.

Bora, E., Yucel, M., \& Pantelis, C. (2009). Cognitive endophenotypes of bipolar disorder: a meta-analysis of neuropsychological deficits in euthymic patients and their first-degree relatives. Journal of Affective Disorders, 113, 1-20.

Bora, E., Yücel, M., \& Pantelis, C. (2010). Cognitive endophenotypes of bipolar disorder: a meta-analysis of neuropsychological deficits in euthymic patients and their first-degree relatives. Journal of Affective Disorders, 127(1-3), 1-9.

Borges, L. J., Trentini, M. C., Bandeira, R. D., \& DellÁglio, D. D. (2008). Neuropsychological assessment of psychological disorders in childhood: a review study. Psico-USF, 13(1), 125-133.

Brooks, J. O., 3rd, Bearden, C. E., Hoblyn, J. C., Woodard, S. A., \& Ketter, T. A. (2010). Prefrontal and paralimbic metabolic dysregulation related to sustained attention in euthymic older adults with bipolar disorder. Bipolar Disorder, 12(8), 866-874.

Brotman, M. A., Rooney, M. H., Skup, M., Pine, D. S., \& Leibenluft, E., (2009). Increased intrasubject variability in response time in youths with bipolar disorder and at-risk family members. Journal of the American Academy of Child and Adolescent Psychiatry, 48, 628-635.

Burdick, K.E., Gunawardane, N., Goldberg, J. F., Halperin, J. M., Garno, J. L., \& Malhotra, A. K. (2009). Attention and psychomotor functioning in bipolar depression. Psychiatric Research, 166 (2-3), 192-200.

Burdick, K. E., Goldberg, T. E., Cornblatm, B. A., Keefe, R. S., Gopin, C. B., Derosse, P., Braga, R.. J., \& Malhotra, A. K. (2011). The MATRICS consensus cognitive battery in patients with bipolar I disorder. Neuropsychopharmacology, 36(8), 1587-1592.

Caligiuri, M. P., \& Ellwanger, J. (2000). Motor and cognitive aspects of motor retardation indepression. Journal of Affective Disorders, 57, 83-93.

Cavanagh, J. T. O., Van Beck, M., Muir, W., \& Blackwood, D. H. R. (2002). Case-control study of neurocognitive function in euthymic patients with bipolar disorder: an association with mania. British Journal of Psychiatry, 180, 320-326.

Chang, Y. H., Chen, S. L., Lee, S. Y., Hsu, Y. W., Wu, J. Y., Chen, S. H., Chu, C. H., Lee, I. H., Yeh, T. L., Tzeng, N. S., Huang, S. Y., Yang, Y. K., \& Lu, R. B. (2012). Neuropsychological functions in bipolar disorders I and II with and without comorbid alcohol dependence. Progress in Neuropsychopharmacology and Biological Psychiatry, 37(2), 211-216.

Chaves, O. C., Lombardo, L. E., Bearden, C. E., Woolsey, M. D., Martinez, D. M., Barrett, J. A., Miller, A. L., Velligan, D. I., \& Glahn, D. C. (2011). Association of clinical symptoms and 
neurocognitive performance in bipolar disorder: a longitudinal study. Bipolar Disorder, 13(1), 118-123.

Chou, Y. H., Wang, S. J., Lirng, J. F., Lin, C. L., Yang, K. C., Chen, C. K., Yeh, C. B., \& Liao, M. H. (2012). Impaired cognition in bipolar I disorder: the roles of the serotonin transporter and brain-derived neurotrophic factor. Journal of Affective Disorders, 143(1-3), 131137.

Clark, L., \& Goodwin, G. M. (2004). State- and trait-related deficits in sustained attention in bipolar disorder. European Archives of Psychiatry and Clinical Neuroscience, 254(2), 61-68.

Clark, L., Iversen, S. D., \& Goodwin, G. M. (2002). Sustained attention deficit in bipolar disorder. Brazilian Journal of Psychiatry, 180, 313-319.

Conners, C. K. (2000). Conners' Continuous Performance Test II: computer program for Windows-Technical guide and software manual. North Tonawanda, NY: Multi-Health Systems.

Coull, J. T., Frith, C. D., Frackowiak, R. S., \& Grasby, P. M. (1996). A fronto-parietal network for rapid visual information processing: a PET study of sustained attention and working memory. Neuropsychologia, 34(11), 1085-1095.

Cuesta, M. J., Pino, O., Guilera, G., Rojo, J. E., Gómez-Benito, J., Purdon, S. E., Franco, M., Martínez-Arán, A., Segarra, N., TabarésSeisdedos, R., Vieta, E., Bernardo, M., Crespo-Facorro, B., Mesa, F., \& Rejas, J. (2011). Brief cognitive assessment instruments in schizophrenia and bipolar patients, and healthy control subjects: a comparison study between the Brief Cognitive Assessment Tool for Schizophrenia (B-CATS) and the Screen for Cognitive Impairment in Psychiatry (SCIP). Schizophrenia Research, 130(1-3), 137-142.

Cummings, E., Donohoe, G., Hargreaves, A., Moore, S., Fahey, C., Dinan, T. G., McDonald, C., O'Callaghan, E., O’Neill, F. A., Waddington, J. L., Murphy, K. C., Morris, D. W., Gill, M., \& Corvin, A. (2013). Mood congruent psychotic symptoms and specific cognitive deficits in carriers of the novel schizophrenia risk variant at MIR-137. Neuroscience Letters, 532, 33-38.

Daban, C., Martinez-Aran, A., Torrent, C., Tabarés-Seisdedos, R., Balanzá-Martínez, V., Salazar-Fraile, J., Selva-Vera, G., \& Vieta, E. (2006). Specificity of cognitive deficits in bipolar disorder versus schizophrenia: a systematic review. Psychotherapy and Psychosomatics, 75(2), 72-84.

Deckersbach, T., McMurrich, S., Ogutha, J., Savage, C. R., Sachs, G., \& Rauch, S. L. (2004). Characteristics of non-verbal memory impairment in bipolar disorder: the role of encoding strategies. Psychological Medicine, 34, 823-832.

Dickerson, F., Stallings, C., Vaughan, C., Origoni, A., Khushalani, S., Dickinson, D., \& Medoff, D. (2011). Cognitive functioning in recent onset psychosis. Journal of Nervous and Mental Disease, 199(6), 367-371.

Doğanavşargil Baysal, G. O., Gökmen, Z., Akbaş, H., Cinemre, B., Metin, O., \& Karaman, T. (2013). Association of serum homocysteine and methionine levels with cognition and functioning in bipolar disorder. Turk Psikiyatri Dergisi, 24(1), 7-16.

Donohoe, G., Duignan, A., Hargreaves, A., Morris, D. W., Rose, E., Robertson, D., Cummings, E., Moore, S., Gill, M., \& Corvin, A. (2012). Social cognition in bipolar disorder versus schizophrenia: comparability in mental state decoding deficits. Bipolar Disorder, 14(7), 743-748.

Elshahawi, H. H., Essawi, H., Rabie, M. A., Mansour, M., Beshry, Z. A., \& Mansour, A. N. (2011). Cognitive functions among euthymic bipolar I patients after a single manic episode versus recurrent episodes. Journal of affective disorders, 130(1-2),180-191.

Evans, J. D., Heaton, R. K., Paulsen, J. S., McAdams, L. A., Heaton, S. C., \& Jeste, D. V. (1999). Schizoaffective disorder: a form of schizophrenia or affective disorder? Journal of Clinical Psychiatry, 60(12), 874-882.

Fleck, D. E., Eliassen, J. C., Durling, M., Lamy, M., Adler, C. M., DelBello, M. P., Shear, P. K., Cerullo, M. A., Lee, J. H., \& Strakowski, S. M. (2012). Functional MRI of sustained attention in bipolar mania. Molecular Psychiatry, 17(3), 325-336.

Frantom, L. V., Allen, D. N., \& Cross, C. L. (2008). Neurocognitive endophenotypes for bipolar disorder. Bipolar Disorder, 10(3), 387399.

Gerber, S. I., Krienke, U. J., Biedermann, N. C., Grunze, H., Yolken, R. H., Dittmann, S., \& Langosch, J. M. (2012). Impaired functioning in euthymic patients with bipolar disorder: HSV-1 as a predictor.
Progress in Neuropsychopharmacology and Biological Psychiatry, 36(1), 110-116.

Gogos, A., Joshua, N., \& Rossell, S. L. (2010). Use of the Repeatable Battery for the Assessment of Neuropsychological Status (RBANS) to investigate group and gender differences in schizophrenia and bipolar disorder. Australian and New Zealand of Journal of Psychiatry, 44(3), 220-229.

Goldberg, T. E., Gold, J. M., Greenberg, R., Griffin, S., Schulz, S.C., Pickar, D., Kleinman, J. E., \& Weinberger, D. R. (1993). Contrasts between patients with affective disorders and patients with schizophrenia on a neuropsychological test battery. American Journal of Psychiatry, 150(9), 1355-1362.

Goodwin, F. K., Jamison, K. R., \& Ghaemi, S. N. (2007). Manicdepressive illness: bipolar disorders and recurrent depression, $2 \mathrm{nd}$ edition. New York: Oxford University Press.

Gottesman, I. I., \& Gould, T. D. (2003). The endophenotype concept in psychiatry: etymology and strategic intentions. American Journal of Psychiatry, 160(4), 636-645.

Grunebaum, H. U., Cohler, B. J., Kauffman, C., \& Gallant, D. (1978). Children of depressed and schizophrenic mothers. Child Psychiatry and Human Development, 8, 219-228.

Gualtieri, C. T., \& Morgan, D. W. (2008). The frequency of cognitive impairment in patients with anxiety, depression, and bipolar disorder: an unaccounted source of variance in clinical trials. Journal of Clinical Psychiatry, 69(7), 1122-1130.

Harkavy-Friedman, J. M., Keilp, J. G., Grunebaum, M. F., Sher, L., Printz, D., Burke, A. K., Mann, J. J., \& Oquendo, M. (2006). Are BPI and BPII suicide attempters distinct neuropsychologically? Journal of Affective Disorders, 94, 255-259.

Hawkins, K. A., Hoffman, R. E., Quinlan, D. M., Rakfeldt, J., Docherty, N. M., \& Sledge, W. H. (1997). Cognition, negative symptoms, and diagnosis: a comparison of schizophrenic, bipolar, and control samples. Journal of Neuropsychiatry Clinical Neurosciences, 9(1), 81-89.

Hellvin, T., Sundet, K., Simonsen, C., Aminoff, S. R., Lagerberg, T. V., Andreassen, O. A., \& Melle, I. (2012). Neurocognitive functioning in patients recently diagnosed with bipolar disorder. Bipolar Disorder, 14(3), 227-238.

Hill, S. K., Reilly, J. L., Harris, M. S., Rosen, C., Marvin, R. W., Deleon, O., \& Sweeney, J. A. (2009). A comparison of neuropsychological dysfunction in first-episode psychosis patients with unipolar depression, bipolar disorder, and schizophrenia. Schizophrenia Research, 113(2-3), 167-175.

Holmes, M. K., Erickson, K., Luckenbaugh, D. A., Drevets, W. C., Bain, E. E., Cannon, D. M., Snow, J., Sahakian, B. J., Manji, H. K., \& Zarate, C. A., Jr. (2008). A comparison of cognitive functioning in medicated and unmedicated subjects with bipolar depression. Bipolar Disorder, 10(7), 806-815.

Honig, A., Arts, B. M., Ponds, R. W., \& Riedel, W. J. (1999). Lithium induced cognitive side effects in bipolar disorder: a qualitative analysis and implications for daily practice. International Clinical Psychopharmacoly, 14, 167-171.

Howells, F. M., Ives-Deliperi, V. L., Horn, N. R., \& Stein, D. J. (2012). Mindfulness based cognitive therapy improves frontal control in bipolar disorder: a pilot EEG study. BMC Psychiatry, 12, 15.

Iverson, G. L., Brooks, B. L., \& Young, A. H. (2009). Rapid computerized assessment of neurocognitive deficits in bipolar disorder. Applied Neuropsychology, 16(3), 207-213.

Iverson, G. L., Brooks, B. L., Langenecker, S. A., \& Young, A. H. (2011). Identifying a cognitive impairment subgroup in adults with mood disorders. Journal of Affective Disorders, 132(3), 360-367.

Jabben, N., Arts, B., Krabbendam, L., \& van Os, J. (2009). Investigating the association between neurocognition and psychosis in bipolar disorder: further evidence for the overlap with schizophrenia. Bipolar Disorder, 11(2), 166-177.

Juselius, S., Kieseppä, T., Kaprio, J., Lönnqvist, J., \& TuulioHenriksson, A. (2009). Executive functioning in twins with bipolar I disorder and healthy co-twins. Archives of Clinical Neuropsychology, 24(6), 599-606.

Kałwa, A. (2011). Cognitive dysfunctions in bipolar disorders. Psychiatr Polska, 45(6), 901-910.

Kempton, M., Geddes, J., Ettinger, U., Williams, S.C., \& Grasby, P. M. (2008). Meta-analysis, database, and metaregression of 98 structural imaging studies in bipolar disorder. Archives of General Psychiatry, 65, 1017-1032. 
Klimes-Dougan, B., Ronsaville, D., Wiggs, E. A., \& Martinez, P. E. (2006). Neuropsychological functioning in adolescent children of mothers with a history of bipolar or major depressive disorders. Biological Psychiatry, 60, 957-965.

Kurnianingsih, Y. A., Kuswanto, C. N., McIntyre, R. S., Qiu, A., Ho, B.C., \& Sim, K. (2011). Neurocognitive-genetic and neuroimaginggenetic research paradigms in schizophrenia and bipolar disorder. Journal of Neural Transmission, 118(11), 1621-1639.

Lahera, G., Pedrera, A., Cabañes, L., Fernandez-Lorente, J., Simal, P., Montes, J. M., \& Saiz-Ruiz, J. (2009). P300 event-related potential in euthymic patients with bipolar disorder. Progress in Neuropsychopharmacology and Biological Psychiatry, 33(1), 1619.

Latalova, K., Jan, P., Tomas, D., Dana, K., \& Hana, V. (2011). Cognitive impairment in bipolar disorder. Biomedical Papers of the Medical Faculty of the University Palacky Olomouc Czech Republic, 155(1), 1926.

Lee, J., Altshuler, M. D., Glahn, D. C., Miklowitz, D. J., Ochsner, K., \& Green, M. F. (2013). Social and nonsocial cognition in bipolar disorder and schizophrenia: relative levels of impairment. American Journal of Psychiatry, 170, 334-341.

Levine, B., Turner, G. R., \& Stuss, D. (2008). Rehabilitation of frontal lobe functions. In D. T. Stuss, G. Winocur, \& I. H. Robertson (Eds.), Cognitive neurorehabilitation: evidence and application, 2nd edition (pp. 464-486). Cambridge, UK: Cambridge University Press.

Levy, B. (2013). Autonomic nervous system arousal and cognitive functioning in bipolar disorder. Bipolar Disorder, 15(1), 70-79.

Levy, B., Stephansky, M. R., Dobie, K. C., Monzani, B. A., Medina, A. M., \& Weiss, R. D. (2009). The duration of inpatient admission predicts cognitive functioning at discharge in patients with bipolar disorder. Comprehensive Psychiatry, 50(4), 322-326.

Lewandowski, K. E., Cohen, B. M., Keshavan, M. S., \& Ongür, D. (2011). Relationship of neurocognitive deficits to diagnosis and symptoms across affective and non-affective psychoses. Schizophrenia Research, 133(1-3), 212-217.

Li, C. T., Hsieh, J. C., Wang, S. J., Yang, B. H., Bai, Y. M., Lin, W. C., Lan, C. C., \& Su, T. P. (2012). Differential relations between fronto-limbic metabolism and executive function in patients with remitted bipolar I and bipolar II disorder. Bipolar Disorder, 14(8), 831-842.

Liu, J. X., Chen, Y. S., Hsieh, J. C., Su, T. P., Yeh, T. C., \& Chen, L. F. (2012). Differences in white matter abnormalities between bipolar I and II disorders. Journal of Affective Disorders, 127(1-3), 309-315.

López-Jaramillo, C., Lopera-Vásquez, J., Gallo, A., Ospina-Duque, J., Bell, V., Torrent, C., Martínez-Arán, A., \& Vieta, E. (2010a). Effects of recurrence on the cognitive performance of patients with bipolar I disorder: implications for relapse prevention and treatment adherence. Bipolar Disorder, 12(5), 557-567.

López-Jaramillo, C., Lopera-Vásquez, J., Ospina-Duque, J., García, J., Gallo, A., Cortez, V., Palacio, C., Torrent, C., MartínezArán, A., \& Vieta, E. (2010b). Lithium treatment effects on the neuropsychological functioning of patients with bipolar I disorder. Journal of Clinical Psychiatry, 71(8), 1055-1060.

Maalouf, F. T., Klein, C., Clark, L., Sahakian, B. J., Labarbara, E. J., Versace, A., Hassel, S., Almeida, J. R., \& Phillips, M. L. (2010). Impaired sustained attention and executive dysfunction: bipolar disorder versus depression-specific markers of affective disorders. Neuropsychologia, 48(6), 1862-1868.

Macqueen, G., \& Young, T. (2003). Cognitive effects of atypical antipsychotics: focus on bipolar spectrum disorders. Bipolar Disorder, 5(Suppl. 2), 53-61.

Mahlberg, R., Adli, M., Bschor, T., \& Kienast, T. (2008). Age effects on trail making test during acute depressive and manic episode. International Journal of Neuroscience, 118(9), 1347-1356.

Malloy-Diniz, L. F., Neves, F. S., Abrantes, S. S., Fuentes, D., \& Corrêa, H. (2009). Suicide behavior and neuropsychological assessment of type I bipolar patients. Journal of Affective Disorders, $112(1-3), 231-236$

Manly, T., \& Robertson, I. H. (1997). Sustained attention and the frontal lobes. In P. Rabbit (Ed.), Methodology of frontal and executive function (pp. 135-153). Hove: Psychology Press.

Marshall, D. F., Walker, S. J., Ryan, K. A., Kamali, M., Saunders, E. F., Weldon, A. L., Adams, K. M., McInnis, M. G., \& Langenecker,
S. A. (2012). Greater executive and visual memory dysfunction in comorbid bipolar disorder and substance use disorder. Psychiatric Research, 200(2-3), 252-257.

Martinez-Aran, A., Scott, J., Colom, F., Torrent, C., TabaresSeisdedos, R., Daban, C., Leboyer, M., Henry, C., Goodwin, G. M., Gonzalez-Pinto, A., Cruz, N., Sanchez-Moreno, J., \& Vieta, E. (2009). Treatment nonadherence and neurocognitive impairment in bipolar disorder. Journal of Clinical Psychiatry, 70(7), 1017-1023.

Martinez-Aran, A., Torrent, C., Tabares-Seisdedos, R., Salamero, M., Daban, C., Balanza-Martinez, V., Sanchez-Moreno, J., Manuel Goikolea, J., Benabarre, A., Colom, F., \& Vieta, E. (2008). Neurocognitive impairment in bipolar patients with and without history of psychosis. Journal of Clinical Psychiatry, 69(2), 233239.

Martino, D. J., Igoa, A., Marengo, E., Scápola, M., \& Strejilevich, S. A. (2011a). Neurocognitive impairments and their relationship with psychosocial functioning in euthymic bipolar II disorder. Journal of Nervous and Mental Disease, 199(7), 459-464.

Martino, D. J., Strejilevich, S. A., Fassi, G., Marengo, E., \& Igoa, A. (2011b). Theory of mind and facial emotion recognition in euthymic bipolar I and bipolar II disorders. Psychiatric Research, 189(3), 379-384

Martino, D. J., Strejilevich, S. A., Scápola, M., Igoa, A., Marengo, E., Ais, E. D., \& Perinot L. (2008). Heterogeneity in cognitive functioning among patients with bipolar disorder. Journal of Affective Disorders, 109(1-2), 149-156.

Martino, D. J., Strejilevich, S. A., Torralva, T., \& Manes, F. (2010). Decision making in euthymic bipolar I and bipolar II disorders. Psychological Medicine, 41, 1319-1327.

McKinnon, M. C., Cusi, A. M., \& MacQueen, G. M. (2012). Psychological factors that may confer risk for bipolar disorder. Cognitive Neuropsychiatry, 18, 115-128.

Mora, E., Portella, M. J., Forcada, I., Vieta, E., \& Mur, M. (2012). Persistence of cognitive impairment and its negative impact on psychosocial functioning in lithium-treated, euthymic bipolar patients: a 6-year follow-up study. Psychological Medicine, 43, 1187-1196.

Morisano, D., Wing, V. C., Sacco, K.A., Arenovich, T., \& George, T. P. (2013). Effects of tobacco smoking on neuropsychological function in schizophrenia in comparison to other psychiatric disorders and non-psychiatric controls. American Journal on Addictions, 22(1), 46-53.

Mur, M., Portella, M. J., Martínez-Arán, A., Pifarré, J., \& Vieta, E. (2008a). Long-term stability of cognitive impairment in bipolar disorder: a 2-year follow-up study of lithium-treated euthymic bipolar patients. Journal of Clinical Psychiatry, 69(5), 712-719.

Mur, M., Portella, M. J., Martínez-Arán, A., Pifarré, J., \& Vieta, E. (2008b). Neuropsychological profile in bipolar disorder: a preliminary study of monotherapy lithium-treated euthymic bipolar patients evaluated at a 2-year interval. Acta Psychiatrica Scandinavica, 118(5), 373-381.

Murphy, F. C., \& Sahakian, B.J. (2001). Neuropsychology of bipolar disorder. British Journal of Psychiatry, 178(Suppl. 41), S120-S127.

Murphy, F. C., Sahakian, B. J., Rubinsztein, J. S., Michael, A., Rogers, R. D., Robbins, T. W., \& Paykel, E. S. (1999). Emotional bias and inhibitory control processes in mania and depression. Psychological Medicine, 29(6), 1307-1321.

Normala, I., Abdul, H. A., Azlin, B., Nik, R. N. J., Hazli, Z., \& Shah, S. A. (2010). Executive function and attention Span in euthymic patients with bipolar I disorder. Medical Journal of Malaysia, 65(3), 199-203.

Olvet, D. M., Burdick, K. E., \& Cornblatt, B. A. (2013). Assessing the potential to use neurocognition to predict who is at risk for developing bipolar disorder: a review of the literature. Cognitive Neuropsychiatry, 18(1-2), 129-145.

Pachet, A. K., \& Wisniewski, A. M. (2003). The effects of lithium on cognition: an updated review. Psychopharmacology, 170, 225-234.

Pan, Y. J., Hsieh M. H., \& Liu, S. K. (2011). Visuospatial working memory deficits in remitted patients with bipolar disorder: susceptibility to the effects of GABAergic agonists. Bipolar Disorder, 13(4), 365-376.

Pattanayak, R. D., Sagar R., \& Mehta, M. (2012). Neuropsychological performance in euthymic Indian patients with bipolar disorder 
type I: correlation between quality of life and global functioning. Psychiatry and Clinical Neurosciences, 66(7), 553-563.

Paus, T., Zatorre, R. J., \& Hofle, N. (1997). Time-related changes in neural systems underlying attention and arousal during the performance of an auditory vigilance task. Journal of Cognitive Neuroscience, 9, 392-408

Pompei, F., Jigar, J., Roberto, T., Paolo, G., Katya, R., Kumari, V., \& Frangou, S. (2011). Familial and disease specific abnormalities in the neural correlates of the Stroop Task in Bipolar Disorder. Neuroimage, 56(3), 1677-1684.

Ponsford, J. (2008). Rehabilitation of attention following traumatic brain injury. In D. Stuss, G. Winocur, \& I.H. Robertson (Eds.), Cognitive neurorehabilitation: evidence and application, 2nd edition (pp. 507-520). Cambridge, UK: Cambridge University Press.

Pradhan, B. K., Chakrabarti, S., Nehra, R., \& Mankotia, A. (2008). Cognitive functions in bipolar affective disorder and schizophrenia: comparison. Psychiatry and Clinical Neurosciences, 62(5), 515525.

Quaishi S., \& Frangou, S. (2002). Neuropsychology of bipolar disorder: a review. Journal of Affective Disorders, 72(3), 209-226.

Rocca, C. C., \& Lafer, B. (2006). Neuropsychological disturbances in bipolar disorder. Revista Brasileira de Psiquiatria, 28(3), 226-237.

Roiser, J. P., Cannon, D. M., Gandhi, S. K., Taylor Tavares, J., Erickson, K., Wood, S., Klaver, J. M., Clark, L., Zarate, C. A., Jr., Sahakian, B. J., \& Drevets, W. C. (2009). Hot and cold cognition in unmedicated depressed subjects with bipolar disorder. Bipolar Disorder, 11(2), 178-189.

Sachs, G., Schaffer, M., \& Winklbaur, B. (2007). Cognitive deficits in bipolar disorder. Neuropsychiatrie, 21(2), 93-101.

Sanchez-Moreno, J., Martinez-Aran, A., Colom, F., Scott, J., TabaresSeisdedos, R., Sugranyes, G., Torrent, C., Daban, C., Benabarre, A., Goikolea, J. M., Franco, C., González-Pinto, A., Ayuso-Mateos, J. L., \& Vieta, E. (2009). Neurocognitive dysfunctions in euthymic bipolar patients with and without prior history of alcohol use. Journal of Clinical Psychiatry, 70(8), 1120-1127.

Sánchez-Morla, E. M., Barabash, A., Martínez-Vizcaíno, V., TabarésSeisdedos, R., Balanzá-Martínez, V., Cabranes-Díaz, J. A., BacaBaldomero, E., \& Gómez, J. L. (2009). Comparative study of neurocognitive function in euthymic bipolar patients and stabilized schizophrenic patients. Psychiatric Research, 169(3), 220-228.

Savitz, J. B., Solms, M., \& Ramesar R. S. (2005). Neurocognitive function as an endophenotype for genetic studies of bipolar affective disorder. Neuromolecular Medicine, 7(4), 275-286.

Sax, K. W., Strakowski, S. M., Zimmerman, M. E., DelBello, M. P., Keck, P. E., Jr., \& Hawkins, J. M. (1999). Frontosubcortical neuroanatomy and the continuous performance test in mania. American Journal of Psychiatry, 156, 139-141.

Schretlen ,D. J., Peña, J., Aretouli, E., Orue, I., Cascella, N. G., Pearlson, G. D., \& Ojeda, N.(2013). Confirmatory factor analysis reveals a latent cognitive structure common to bipolar disorder, schizophrenia, and normal controls. Bipolar Disorder, May 9.

Selva, G., Salazar, J., Balanza-Martinez, V., Martinez-Aran, A., Rubio, C., Daban, C., Sanchez-Moreno, J., Vieta, E., \& Tabares-Seisdedos, R. (2007). Bipolar I patients with and without a history of psychotic symptoms: do they differ in their cognitive functioning? Journal of Psychiatric Research, 41, 265-272.

Sepede, G., De Berardis, D., Campanella, D., Perrucci, M. G., Ferretti, A., Serroni, N., Moschetta, F. S., Del Gratta, C., Salemo, R. M., Ferro, F. M., Di Giannantonio, M., Onofrj, M., Romani, G. L., \& Gambi, F. (2012). Impaired sustained attention in euthymic bipolar disorder patients and non-affected relatives: an fMRI study. Bipolar Disorder, 14(7), 764-779.

Shan, C., Lee, S. Y., Chang, Y. H., Wu, J. Y., Chen, S. L., Chen, S. H., Hsiao, Y. L., Yang, H. F., Lee, I. H., Chen, P. S., Yeh, T. L., Yang, Y. K., \& Lu, R. B. (2011). Neuropsychological functions in Han Chinese patients in Taiwan with bipolar II disorder comorbid and not comorbid with alcohol abuse/alcohol dependence disorder. Progress in Neuropsychopharmacology and Biological Psychiatry, 35(1), 131-136.

Simonsen, C., Sundet, K., Vaskinn, A., Birkenaes, A. B., Engh, J. A., Hansen, C. F., Jónsdóttir, H., Ringen, P. A., Opjordsmoen, S., Friis, S., \& Andreassen, O. A. (2008). Neurocognitive profiles in bipolar
I and bipolar II disorder: differences in pattern and magnitude of dysfunction. Bipolar Disorder, 10(2), 245-255.

Soeiro-de-Souza, M. G., Bio, D. S., Dias, V. V., Vieta, E., MachadoVieira, R., \& Moreno, R. A. (2013). The CACNA1C risk allele selectively impacts on executive function in bipolar type I disorder. Acta Psychiatica Scandinavica, in press.

Soeiro-de-Souza, M. G., Machado-Vieira, R., Soares Bio, D., Do Prado, C. M., \& Moreno, R. A. (2012). COMT polymorphisms as predictors of cognitive dysfunction during manic and mixed episodes in bipolar I disorder. Bipolar Disorder, 14(5), 554-564.

Soeiro-de-Souza, M. G., Post, R. M., de Sousa, M. L., Missio, G., do Prado, C. M., Gattaz, W. F., Moreno, R. A., \& Machado-Vieira, R. (2012). Does BDNF genotype influence creative output in bipolar I manic patients? Journal of Affective Disorders, 139(2), 181-186.

Solé, B., Bonnin, C. M., Torrent, C., Balanzá-Martínez, V., TabarésSeisdedos, R., Popovic, D., Martínez-Arán, A., \& Vieta, E. (2012). Neurocognitive impairment and psychosocial functioning in bipolar II disorder. Acta Psychiatrica Scandinavica, 125(4), 309-317.

Stoll, A. L., Renshaw, P. F., Yurgelun-Todd, D. A., \& Cohen, B. M. (2000). Neuroimaging in bipolar disorder: what have we learned? Biological Psychiatry, 48(6), 505-517.

Strakowski, S. M., Fleck, D. E., DelBello, M. P., Adler, C. M., Shear, P. K., McElroy, S. L., Keck, P. E., Jr., Moss, Q., Cerullo, M. A., Kotwal, R., \& Arndt, S. (2009). Characterizing impulsivity in mania. Bipolar Disorder, 11(1), 41-51.

Strakowski, S. M., Fleck, D. E., DelBello, M. P., Adler, C. M., Shear, P. K., Kotwal, R., \& Arndt, S. (2010). Impulsivity across the course of bipolar disorder. Bipolar Disorder, 12(3), 285-297.

Strauss, M. E., Bohannon, W. E., Stephens, J. H., \& Pauker, N. E. (1984) Perceptual Span in schizophrenia and affective disorders. Journal of Nervous and Mental Disease, 172, 431-435.

Swann, A. C., Lijffijt, M., Lane, S. D., Steinberg, J. L., \& Moeller, F. G. (2009). Severity of bipolar disorder is associated with impairment of response inhibition. Journal of Affective Disorders, 116(1-2), 30-36.

Tabarés-Seisdedos, R., Balanzá-Martínez, V., Sánchez-Moreno, J., Martinez-Aran, A., Salazar-Fraile, J., Selva-Vera, G., Rubio, C., Mata, I., Gomez-Beneyto, M., \& Vieta, E. (2008). Neurocognitive and clinical predictors of functional outcome in patients with schizophrenia and bipolar I disorder at one-year follow-up. Journal of Affective Disorders, 109(3), 286-299.

Thompson, J. M., Gallagher, P., Hughes, J. H., Watson, S., Gray, J. M., Ferrier, I. N., \& Young, A. H. (2005). Neurocognitive impairment in euthymic patients with bipolar affective disorder. Brazilian Journal of Psychiatry, 186, 32-40.

Thompson, J. M., Gray, J. M., Crawford, J. R., Hughes, J. H., Young, A. H., \& Ferrier, I. N. (2009). Differential deficit in executive control in euthymic bipolar disorder. Journal of Abnormal Psychology, $118(1), 146-160$

Torralva T., Gleichgerrcht E., Torrente F., Roca M., Strejilevich, S. A., Cetkovich, M., Lischinsky, A., \& Manes, F. (2011). Neuropsychological functioning in adult bipolar disorder and ADHD patients: a comparative study. Psychiatric Research, 186(23), 261-266.

Torrent, C., Martinez-Arán, A., Daban, C., Amann, B., BalanzáMartínez, V., del Mar Bonnín, C., Cruz, N., Franco, C., TabaresSeisdedos, R., \& Vieta, E. (2011). Effects of atypical antipsychotics on neurocognition in euthymic bipolar patients. Comprehensive Psychiatry, 52(6), 613-622.

Torrent, C., Martinez-Arán, A., del Mar Bonnin, C., Reinares, M., Daban, C., Solé, B., Rosa, A. R., Tabares-Seisdedos, R., Popovic, D., Salamero, M., \& Vieta, E. (2012). Long-term outcome of cognitive impairment in bipolar disorder. Journal of Clinical Psychiatry, 73(7), e899-e905.

Torres, I. J., DeFreitas, V. G., DeFreitas, C. M., Kauer-Sant'Anna, M., Bond, D. J., Honer, W. G., Lam, R. W., \& Yatham, L. N. (2010). Neurocognitive functioning in patients with bipolar I disorder recently recovered from a first manic episode. Journal of Clinical Psychiatry, 71(9), 1234-1242.

Trivedi, J. K., Goel, D., Sharma, S., Singh, P. K., \& Tandon, R. (2008) Cognitive functions in stable schizophrenia \& euthymic state of bipolar disorder. Indian Journal of Medicine Research, 126(5), 433-439. 
Tuulio-Henriksson, A., Perälä, J., Saarni, S. I., Isometsä, E., Koskinen, S., Lönnqvist, J., \& Suvisaari, J. (2011). Cognitive functioning in severe psychiatric disorders: a general population study. European Archives of Psychiatry and Clinical Neuroscience, 261(6), 447-456.

Van der Werf-Eldering, M. J., Burger, H., Holthausen, E. A., Aleman, A., \& Nolen, W. A. (2010). Cognitive functioning in patients with bipolar disorder: association with depressive symptoms and alcohol use. PLoS One, 5(9), e13032.

Van der Werf-Eldering, M. J., Burger, H., Jabben, N., Holthausen, E. A., Aleman, A., \& Nolen, W. A. (2011). Is the lack of association between cognitive complaints and objective cognitive functioning in patients with bipolar disorder moderated by depressive symptoms? Journal of Affective Disorders, 130(1-2), 306-311.

Van Gorp, W. G., Altshuler, L., Theberge, D. C., Wilkins, J., \& Dixon, W. (1998). Cognitive impairment in euthymic bipolar patients with and without prior alcohol dependence. Archives of General Psychiatry, 55(1), 41-46.

Vaskinn, A., Sundet, K., Simonsen, C., Hellvin, T., Melle, I., \& Andreassen, O. A. (2011). Sex differences in neuropsychological performance and social functioning in schizophrenia and bipolar disorder. Neuropsychology, 25(4), 499-510.

Vierck, E., Porter, R. J., Luty, S. E., Moor, S., Crowe, M. T., Carter, J. D., Inder, M. L., \& Joyce, P. R. (2013). Further evidence for slow binocular rivalry rate as a trait marker for bipolar disorder. Australian and New Zealand Journal of Psychiatry, 47(4), 371-379.

Vieta, E. (2012). Avanços no transtorno bipolar. Recife: Conectfarma.

Watson, S., Gallagher, P., Porter, R. J., Smith, M. S., Herron, L. J., Bulmer, S. North-East Mood Disorders Clinical Research Group, Young, A. H., \& Ferrier, I. N. (2012). A randomized trial to examine the effect of mifepristone on neuropsychological performance and mood in patients with bipolar depression. Biological Psychiatry ,72(11), 943-949.

Wobrock, T., Ecker, U. K., Scherk, H., Schneider-Axmann, T., Falkai, P., \& Gruber, O. (2009). Cognitive impairment of executive function as a core symptom of schizophrenia. World Journal of Biological Psychiatry, 10, 442-451.

Wu, H. I., Chang, Y. H., Lai, C. C., Wu, J. Y., Chen, S. L., Chu, C. H., Lee, I. H., Yeh, T. L., Tzeng, N. S., Huang, S. Y., Yang, Y. K., \& Lu, R. B. (2011). The effect of comorbid anxiety disorder on neuropsychological function in bipolar II disorder. Progress in Neuropsychopharmacology and Biological Psychiatry, 35(8), 1841-1845.

Xu, G., Lin, K., Rao, D., Dang, Y., Ouyang, H., Guo, Y., Ma, J., \& Chen, J. (2012). Neuropsychological performance in bipolar I, bipolar II and unipolar depression patients: a longitudinal, naturalistic study. Journal of Affective Disorders, 136(3), 328339.

Yates, D. B., Dittmann, S., Kapczinski, F., \& Trentini, C. M. (2011). Cognitive abilities and clinical variables in bipolar I depressed and euthymic patients and controls. Journal of Psychiatric Research, 45(4), 495-504.

Yoram, B., Galit, E., Tal, S., Hila, G. Z., Eiran Vadim, H., Yuval, B., \& Yechiel, L. (2013). A longitudinal study of cognition in asymptomatic and mildly symptomatic bipolar disorder patients. Psychiatric Research, in press.

Yurgelun-Todd, D., Shi, L., Zhu, B., Namjoshi, M., Tunis, S., Baker, R. W., \& Tohen, M. (2002). Olanzapine vs divalproex: prospective comparison on self-reported cognitive function in patients with bipolar disorder. European Neuropsychopharmacology, 12(Suppl. 3), 308-309.

Zalla, T., Joyce, C., Szoke, A., Schurhoff, F., Pillon, B., Komano, O., Perez-Diaz, F., Bellivier, F., Alter, C., Dubois, B., Rouillon, F., Houde, O., \& Loboyer, M. (2004). Executive dysfunctions as potential markers off amilial vulnerability to bipolar disorder and schizophrenia. Psychiatric Research, 121, 207-217.

Zubieta, J. K., Huguelet, P., O’Neil, R. L., \& Giordani, B. J. (2001). Cognitive function in euthymic bipolar I disorder. Psychiatric Research, 102, 9-20. 